\title{
ESTRUCTURA GEOLÓGICA DEL VALLE DE LA CIUDAD DE GUATEMALA INTERPRETADA MEDIANTE UN MODELO DE CUENCA POR DISTENSIÓN
}

\author{
GEOLOGICAL STRUCTURE OF GUATEMALA CITY VALLEY INTERPRETED \\ AS A PULL APART BASIN
}

\author{
Carlos L. Pérez \\ Ingeniería Geotécnica S. A., \\ $1^{\text {a }}$ calle A 15 - 14 zona 8, Pinares, San Cristobal, Mixco 01057 \\ ingenieria-geotecnica@hotmail.com
}

(Recibido: 02/06/09; aceptado:12/12/09)

\begin{abstract}
A new model of Guatemala City Valley geological structure is presented. The Valley was formed by tectonic and volcanic events related to an incipient pull apart basin. This interpretation is supported by geological, geomorhological and seismic evidence. Since Middle Miocene, considerable activity took place along the Motagua and Jalpatagua faults, fracturing the crust and allowing magma to rise and to form several volcanic structures presently in place along the Guatemala City Valley. The activity continued until Middle Pliocene, when the last volcanic center, the Pinula Volcano, was developed in the west side of the Valley. The extension zone originated the Mixco Fault, a normal structure with a $500 \mathrm{~m}$ vertical displacement, affecting even the present volcanic landscape. Basins formed along the fault have been filled with thick pyroclastic deposits. Tectonic activity within the Guatemala Valley is represented only by the Mixco Fault System, that includes several concentric structures. The western edge of the volcanic relict is the El Trébol Fault.

Key words: Guatemala, pull apart basin, Motagua Fault, Jalpatagua Fault, Mixo Fault.

RESUMEN: Se presenta un nuevo modelo que interpreta la estructura geológica del valle de la ciudad de Guatemala, en el que se considera que el valle se ha formado como una estructura de pull apart basin, que se encuentra en una etapa incipiente. Durante su formación han interactuado eventos tectónicos y volcánicos. La interpretación se respalda en evidencias geológico- geomorfológicas y sísmicas. En el Mioceno Medio, se inicia una considerable actividad en las fallas de Motagua y Jalpatagua, que provocan la fractura de la corteza, permitiendo el ascenso de magmas que forman varias estructuras volcánicas en el lugar, donde actualmente está el valle de la ciudad de Guatemala. La actividad continúa hasta el Plioceno Medio, período en el cual se considera que ya se habían desarrollado el centro volcánico Pinula, actualmente al W del valle. Al ampliarse la zona de distensión, se desarrolla la falla Mixco, que afecta el paisaje volcánico hasta la actualidad, una falla normal, con un desplazamiento vertical de mas de $500 \mathrm{~m}$, originando depresiones que se han rellenado de un potente espesor de piroclastos, depositados durante varios eventos sucesivos. La actividad tectónica dentro del valle de Guatemala, está representada únicamente por el sistema de fallas de Mixco, que tienen una forma semicircular concéntrica. El límite W del relicto volcánico es la falla El Trébol.

Palabras clave: Guatemala, pull apart basin, falla Motagua, falla Jalpatagua, falla Mixo.
\end{abstract}

PÉREZ, C.L., 2009: Estructura geológica del valle de la ciudad de Guatemala interpretada mediante un modelo de cuenca por distensión.- Rev. Geól. Amér. Central, 41: 71-78. 


\section{INTRODUCCIÓN}

A la luz de recientes investigaciones geológicas realizadas en el área de San José Pinula (Pérez, 2009), se resalta la existencia de una estructura volcánica que no se ajusta al modelo que interpreta que la estructura del valle de Guatemala, es de naturaleza tectónica y fue definida por G. Dengo, en 1970, como un graben (Weyl, 1,980; Bonis, 1978; Bonis, 1993). Los datos que respaldan la presente interpretación denotan la existencia de dos relictos volcánicos de edad Terciaria que subyacen dentro del relleno de piroclastos. En este artículo se presentan las principales características del modelo tradicional, llamado Modelo Tectónico y a continuación se presentan las características del nuevo modelo, resaltando las estructuras que con el modelo Tectónico no se pueden explicar.

\section{ANTECEDENTES}

Las rocas volcánicas del Terciario en Guatemala, cubren una franja, que se encuentra entre la sierra de Chuacuz y la actual cordillera volcánica del Cuaternario. No existe mucha investigación geológica en esta región, por lo que Sapper, en1937 (en: Weyl, 1980) expresó que el conocimiento del vulcanismo del Terciario de Guatemala es insuficiente. Por otra parte, Williams (en: Weyl, 1980) expresa que estos materiales han sido producto de erupciones por fisuras, resaltando que este tipo de erupciones también son típicas del Terciario en México y Estados Unidos. Dengo (1978) en la reseña sobre el marco tectónico de la región del Caribe, indica que aún quedan aspectos que resolver con respecto a los fenómenos intraplaca, resaltando dentro de éstos, la formación del graben de la Ciudad de Guatemala.

En la cartografía geológica existente 1:250 000 (Bonis, 1993), se destacan las estructuras volcánicas del Cuaternario, que incluyen los conos volcánicos, el área de influencia de los productos lávicos y abanicos asociados a las estructura. En contraste, los productos del vulcanismo Terciario se presentan como amplias áreas en las que tanto la estructura como los productos tienen una escasa o ausente delimitación o interpretación geológica (Fig. 1).

\section{MODELO TECTÓNICO DE GRABEN}

Se ha aceptado por la comunidad geocientífica, que la estructura del valle de la ciudad de Guatemala es una depresión de origen tectónico, tipo graben (Fig 1), delimitada por las fallas de Mixco y Pinula (Weyl 1980; Plafker, 1978; Bonis, 1978; Bonis, 1993); de acuerdo al relieve actual, el desnivel entre los bloques levantados y el graben, donde se encuentra el valle es de $500 \mathrm{~m}$.

En la interpretación de la estructura del graben de la Ciudad de Guatemala, las fallas de Mixco y Pinula, son fallas normales de gran ángulo, aproximadamente paralelas de $20 \mathrm{~km}$ de largo, con una orientación norte a noreste (Fig. 1), que por su actividad han provocado la depresión tectónica que se ha rellenado por materiales procedentes de una serie de eventos de flujos de piroclastos. El graben se encuentra delimitado en el norte por rocas intrusivas y metamórficas del Cretácico, al sur de la falla del Motagua; al sur lo limita la cordillera volcánica del Cuaternario, específicamente por los volcanes de Agua y Pacaya.

\section{MODELO PULL APART BASIN}

\section{Evidencia geológico-geomorfológica}

Durante recientes investigaciones geológicas (Pérez, 2009), se identificó en la parte sureste del valle de Guatemala la presencia de una unidad de sedimentos finos, que se depositaron como flujos de lodo, llenando cañadas y depresiones existentes. Esta Unidad se ha denominado Abanicos Lobulares de Fraijanes y está formada por suelos de grano fino (limo-arcillosos), consolidados, con algunas partículas tamaño grava, interpretados de naturaleza limnica (Fig. 2). La interpretación geomorfológica de la parte alta de la cuenca, revela la existencia de una antigua depresión que se aproxima a una forma circular (Fig. 3); se interpreta que dentro de esta depresión existió un laguna, donde se acumularon sedimentos y al fallar uno de los bordes de la estructura, se produjo la movilización de los sedimentos, transportándose como flujos de lodo, formando la Unidad de Abanicos Lobulares de Fraijanes. 


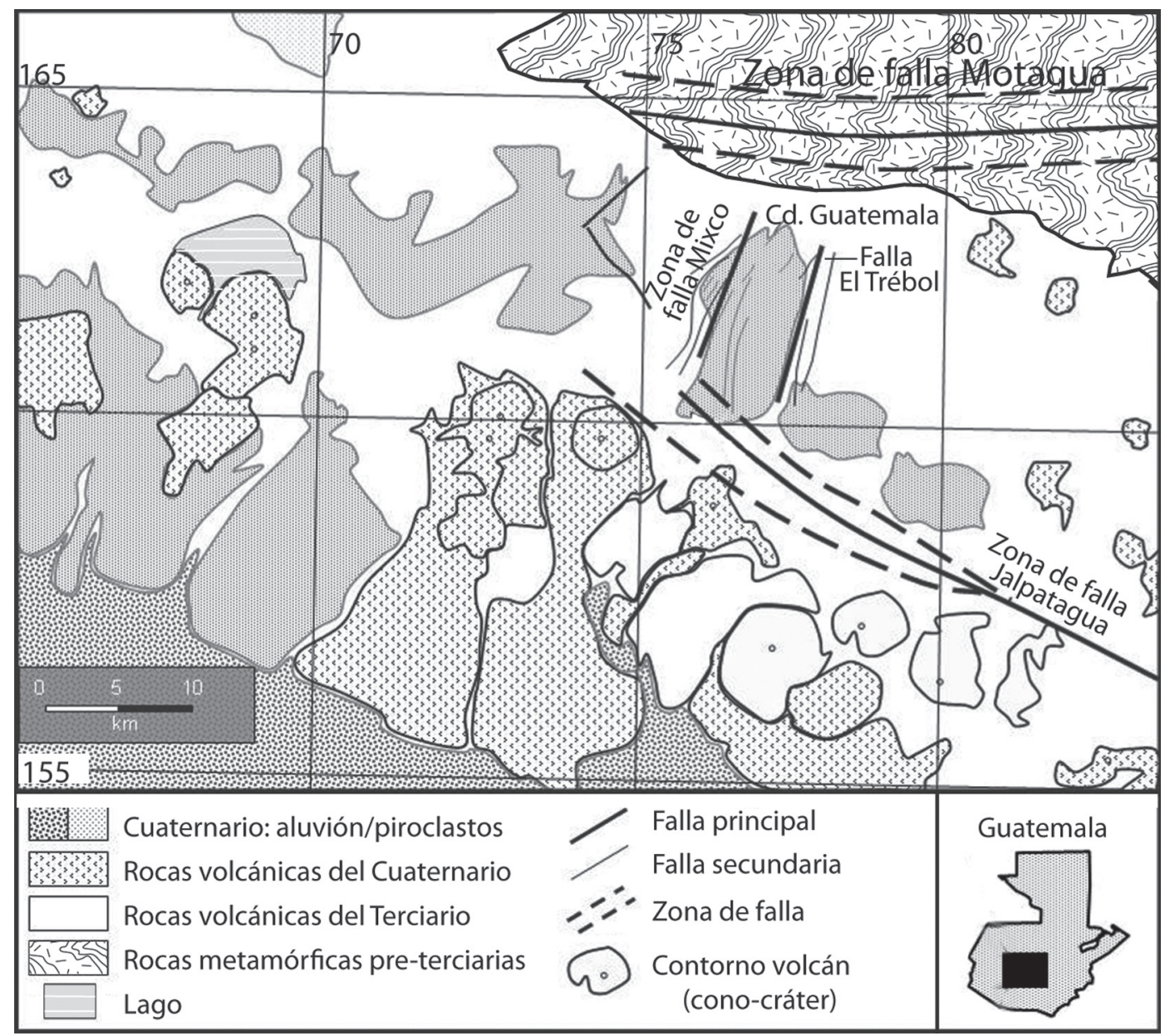

Fig. 1: Mapa geológico, basado en el mapa geológico de Guatemala (escala 1:250 000). Se muestra el modelo tectónico que interpreta la estructura del valle de Guatemala como un graben, limitado al este por la falla de Pinula y al oeste por la falla de Mixco.

Al ampliar la interpretación geomorfológica, se reconocen otras formas concéntricas con el área de la laguna, las cuales se adentran en el valle de la ciudad de Guatemala y el límite coincide con el afloramiento de las rocas volcánicas del Terciario, incluyendo algunos altos estructurales que se encuentran dentro de la ciudad y que por muchos años su interpretación ha sido un enigma (Fig. 3). De acuerdo a los contornos geomorfológicos y su coincidencia con rocas volcánicas, se concluye corresponden con un relicto de un edificio volcánico, que ha sido profundamente erosionado y cubierto por el relleno de piroclastos, el cual ha sido denominado relicto del volcán Pinula.

\section{Evidencia Sísmica}

Durante el terremoto de 1976, la comunidad geocientífica de ese entonces se organizó y aprovechando que las trazas de varias fallas secundarias asociadas a la falla de Mixco eran visibles, se cartografiaron, dando como resultado el Mapa de Fracturas del terremoto de 1976, a escala 1:25000 (SGG, 1976). Analizando la ubicación de las trazas, éstas se presentan desde el centro hasta la parte oeste del Valle, en sectores bien definidos como Ciudad San Cristóbal, Colonia La florida, Colonia San Francisco, Colonia La Florida y teniendo como límite, el 


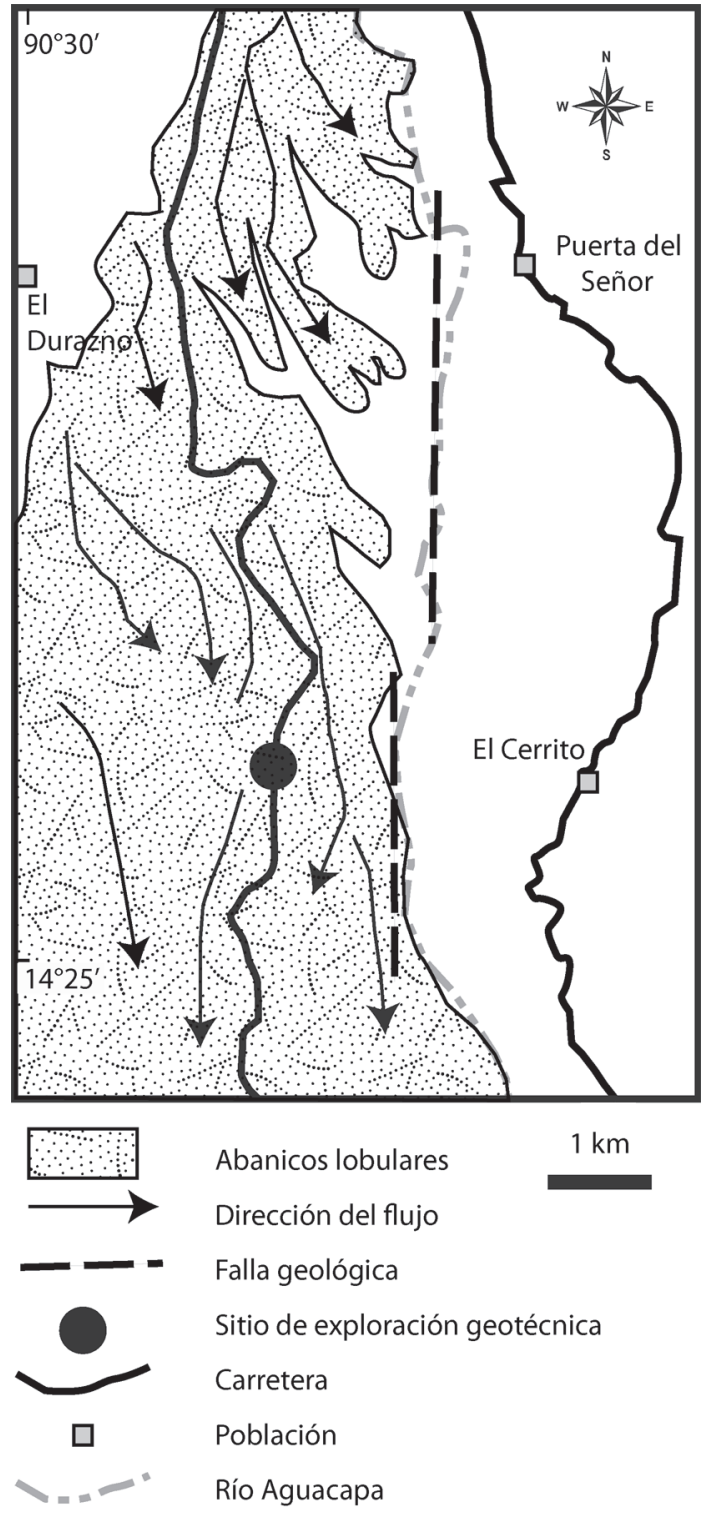

Fig. 2: Mapa geomorfológico, interpretado con base en el mapa de Guatemala (escala 1:50 000), hoja Nueva Santa Rosa.

alineamiento que se encuentra en la parte central del valle y que coincide con El Trébol y se extiende hacia el Norte en el cauce de los río La Barranca y hacia el Sur con el cauce del río Guadroncito. En este mapa, se definen claramente dos sectores, uno es la parte este del valle y el entorno del cerro El Naranjo, donde las fracturas provocadas por el terremoto están ausentes (Fig. 4) y el otro es en el que se concentran las fracturas. Es de resaltar que las fracturas no son lineales, sino que son sectores circulares que se presentan paralelas a los contornos definidos para la parte este del valle de la ciudad de Guatemala.

\section{ESTRUCTURA DEL VALLE DE GUATEMALA}

De acuerdo a estas evidencias, se concluye que bajo el valle de la ciudad de Guatemala ha sido formado como una estructura de tipo pull apart basin, delimitado al norte por la falla del Motagua, al sur por la falla de Jalpatagua y en el centro se ha generado una la zona de distensión que formó la depresión en la que se encuentra la ciudad. La zona de distensión primero fue aprovechada por la actividad volcánica representada por los volcanes Pinula y El Naranjo. Al continuar los movimientos tectónicos colapsaron las estructuras volcánicas formando el sistema de fallas de Mixco, cuyas trazas se evidenciaron en superficie durante el terremoto de 1976 (Fig. 4). Estas estructuras han sido cubiertas por un potente espesor de materiales piroclásticos que han encubierto y ocultado su origen.

De acuerdo a la presente interpretación, el patrón del fracturamiento del valle de Guatemala provocado por el terremoto de 1976 ha evidenciado que la zona de fallamiento coincide únicamente con la falla de Mixco, la cual es un sistema de fallas normales, escalonadas, cuya forma está gobernada por las estructuras volcánicas subyacentes (Fig. 5). Lo que hasta ahora se conoce como falla Pinula, representa las faldas del relicto de la estructura volcánica.

La estructura del relicto volcánico se adentra en el valle de Guatemala hasta el sector de El Trébol, siendo el contorno exterior, el formado por los altos estructurales que se encuentran dentro del valle de Guatemala. Hacia el oeste de este contorno, se inicia la zona de fallamiento activo. 


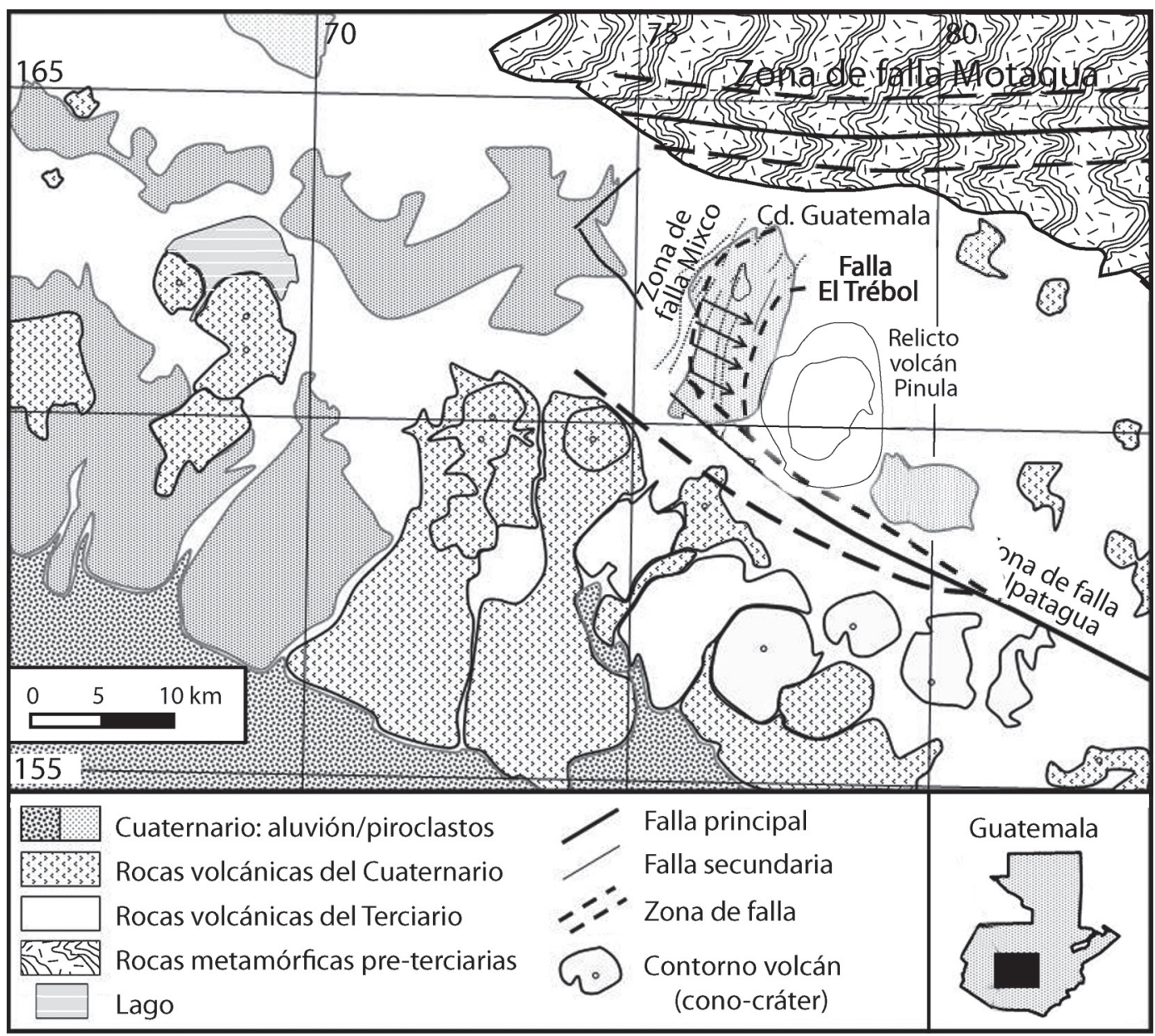

Fig. 3: Modelo tectónico que interpreta la estructura del valle de Guatemala, mediante un pull apart basin, limitado al oeste por la falla de Mixco.

\section{Historia geológica del valle de Guatemala}

El proceso de formación del valle de la ciudad de Guatemala, está relacionado con las fallas transcurrentes de Motagua y Jalpatagua (Fig. 3) y zona de distensión que se forma en intersección de éstas. El incremento en la actividad de las fallas Motagua y Pinula en el Mioceno Medio (Weyl, 1980), creó una zona de debilidad que fue aprovechada para el ascenso de magmas que formaron las estructuras volcánicas de el volcán Pinula y El Naranjo. En un principio esta zona de distensión sirvió como conducto para el ascenso de los magmas, sin embargo, al continuar los movimientos de estas fallas en la zona de distensión, se originó la falla de Mixco cuya forma sigue el contorno de la estructura del relicto del volcán Pinula y que provoca la subsidencia del valle y de las estructuras volcánicas, mediante desplazamientos verticales continuos. El límite entre la depresión y la estructura volcánica está formado por la Falla El Trébol, dentro del valle de la ciudad de Guatemala. El cerro El Naranjo es otra estructura que se encuentra dentro del valle, se considera una 


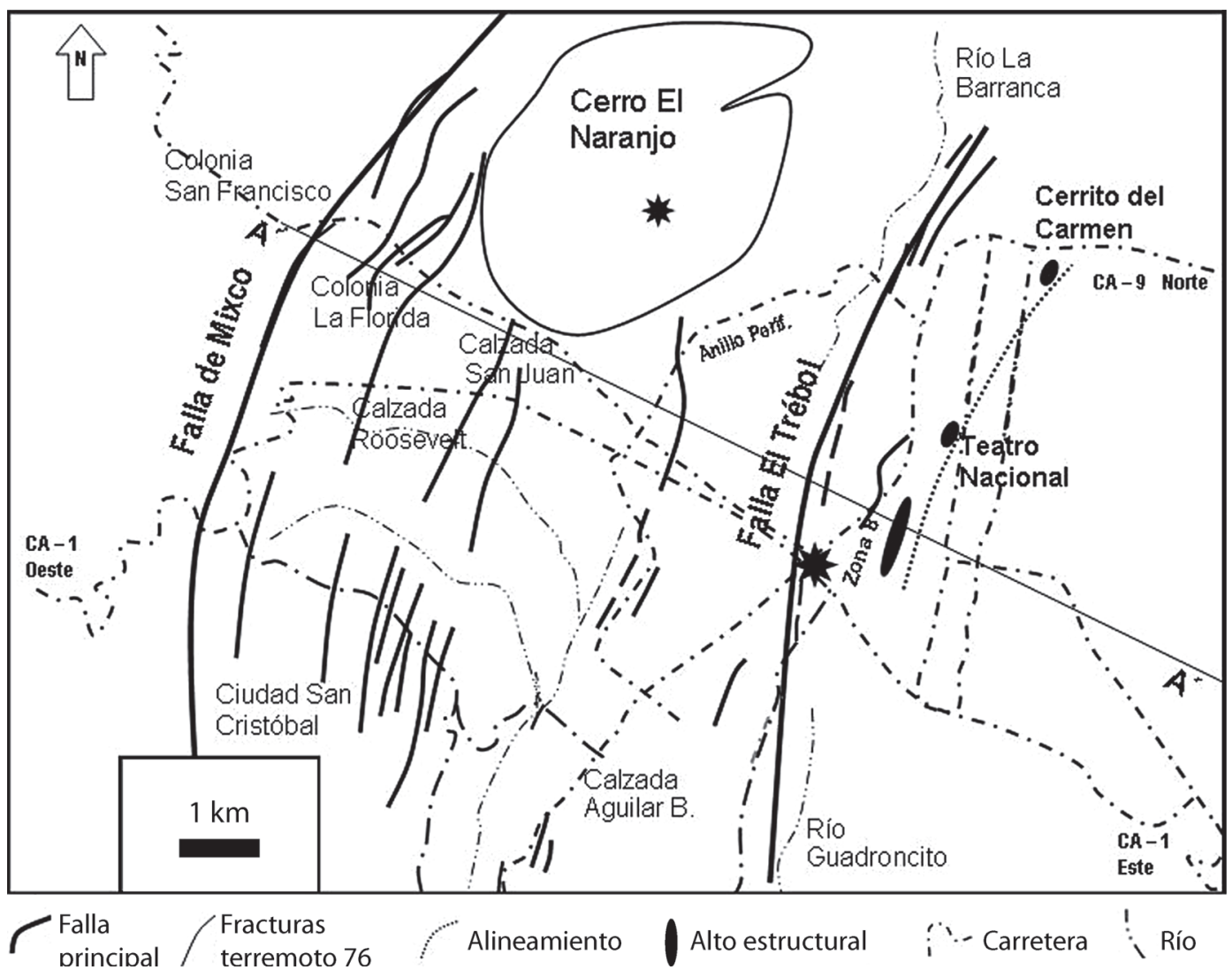

Fig. 4: Mapa de fracturas provocadas por el terremoto de 1976 en el valle de Guatemala (S.G.G, 1,976). Los sectores en los que no se presentan fracturas coinciden con el cerro El Naranjo y el sector E de la ciudad. Nótese la estrella indicando la localidad de El Trébol.

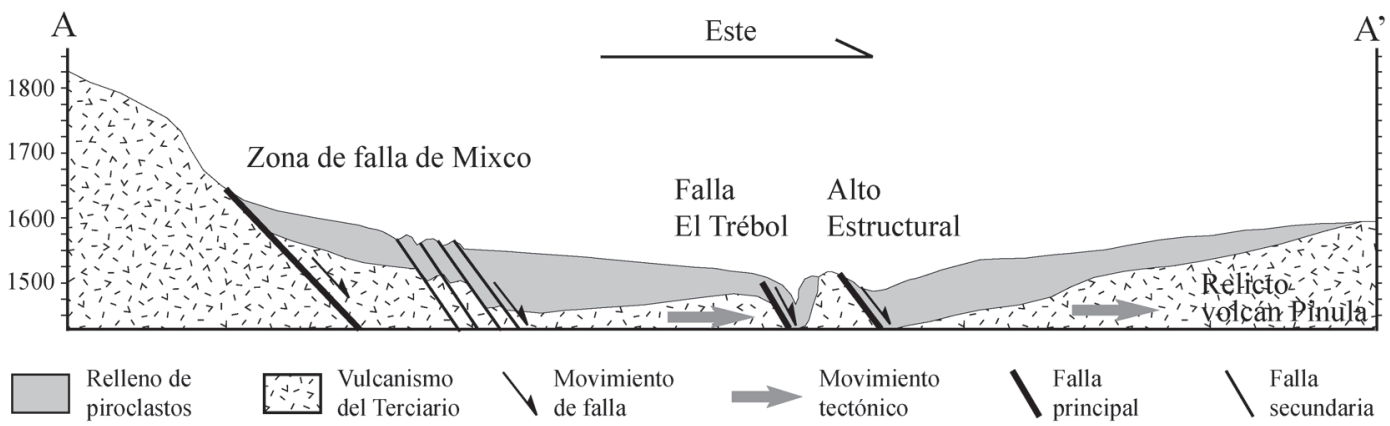

Fig. 5: Sección transversal esquemática del valle de la ciudad de Guatemala con orientación E-W. En esta sección se puede observar que al W el valle está atravesado por el sistema de fallas de Mixco, mientras que en el E se encuentra la falda del relicto del volcán de Pinula. 
estructura secundaria, formada posteriormente al volcán Pinula y que surge de una pequeña cámara magmática, la cual aprovecha la zona de debilidad que coincide con el plano de la falla de Mixco. La actividad volcánica en esta área termina al migrar el vulcanismo hacia la zona de subducción.

\section{CONCLUSIONES}

El modelo que interpreta la estructura del valle de Guatemala como tipo cuenca distensión (pull apart basin), es congruente con el afloramiento de las rocas volcánicas del Terciario y con las fracturas provocadas por el terremoto de 1976. Este modelo establece que la única zona de ruptura en el valle, corresponde con la zona de falla de Mixco, la cual es una serie de fallas normales escalonadas que subyacen el valle de Guatemala, con desplazamiento vertical y que tienen una forma semicircular, gobernada por las estructuras volcánicas.

Mediante este modelo, se interpreta que bajo el relleno de piroclastos del valle de la ciudad de Guatemala, se encuentran subyaciendo dos estructuras volcánicas, las cuales son relictos de los volcanes Pinula y el relicto de El Naranjo.

El presente trabajo se considera un punto de partida para retomar el estudio de la estructura del valle de la ciudad de Guatemala, con una nueva visión, pues aún existen muchas particularidades que se deben de resolver.

\section{AGRADECIMIENTOS}

El autor agradece al Dr. Samuel Bonis por su larga trayectoria, tesonero trabajo en el campo geológico y sus publicaciones coadyuvan al desarrollo del conocimiento científico, al Ing. Carlos Alberto Tobar Jiménez, por ser durante muchos años un apoyo constante y motivador incansable del recurso humano de quienes trabajamos en el campo de las Ciencias de la Tierra, al Geólogo Percy Denyer por su apoyo en la presente publicación y sobre todo a mi familia.

\section{REFERENCIAS}

BONIS, S., 1978: Unheeded geological warnings from 1976, Gutatemala earthquake.- Proc. of Int. Symp. On the February 4th., 1976 Guatemalan earthquake and the reconsturction process. Guatemala, I: Ponencia 9.

BONIS, S. (ed.), 1993: Mapa Geológico de Guatemala, hoja Guatemala [2a ed.].- Escala 1:250,000, IGN, Guatemala.

DENGO, G. 1978: Marco tectónico de la región Caribe.- Proc. of Int. Symp. On the February 4th., 1976 Guatemalan earthquake and the reconsturction process. Guatemala, I: Ponencia 2.

SGG (SOCIEDAD GEOLÓGICA DE GUATEMALA), 1976: Mapa de fracturas, sismos de Febrero de 1976, valle de Guatemala.- Escala 1:25 000, IGN, Guatemala.

PÉREZ, C., 2009: Estudio geotécnico para la planificación de la construcción de la Planta para proceso de alimentos ACSA, San José Pinula, Guatemala.- 24 págs. Ingeniería de Materiales, Guatemala [Inf. interno].

PÉREZ, C., 2009: Estudio geotécnico para la planificación de la ampliación del Centro Educativo Terra Nova, zona 15, Vista Hermosa, Guatemala.- 21 págs. Ingeniería de Materiales, Guatemala [Inf. interno].

PLAFKER, G. 1978: Tectonic significance of suface faulting related to The 4 february 1976 Guatemala earthquake.- Proc. of Int. Symp. On the February 4th., 1,976 Guatemalan earthquake and the reconsturction proces. Guatemala, I: Ponencia 3.

WEYL, R., 1980: Geology of Central America, [2 $2^{\mathrm{a}}$ ed.].- 371 págs. Gebrüder Brontaeger, Stuttgart. 
